\title{
Severe anaphylactic facial edema after botulinum toxin injections for frontal wrinkles: a case report
}

\author{
Chen Zhang, MD, PhD (1D \\ Department of Plastic and Aesthetic Surgery, Xinhua Hospital Affiliated to Dalian University, Dalian, China
}

Botulinum toxin injections are a popular cosmetic procedure because of their satisfactory results, less downtime, and limited side effects. However, some rare adverse events may occur soon after the procedure. Here, we present the case of a patient who developed severe facial edema after her first botulinum toxin injections for frontal wrinkles. A 28-year-old female was injected with 20 units of botulinum toxin to eliminate her frontal and glabella wrinkles. The injection was completed smoothly and uneventfully. Six hours post injection, the patient's face started to swell up and redden with a burning feeling, and the swelling progressively worsened progressively. She was diagnosed with Type I hypersensitivity after the level of immunoglobulin $\mathrm{E}$ in her blood serum was tested. Her symptoms were controlled by treatment with systemic corticosteroids and antihistamines in the next two days and the patient was cured with no recurrence during a 2-year follow-up. This case demonstrates the possibility of developing severe facial edema after botulinum toxin injection. Treatment with systemic corticosteroids and oral antihistamines would help to control this allergic adverse effect.

Keywords: adverse effects; botulinum toxins; cosmetics; edema; face

\section{Introduction}

Botulinum toxin injections are a popular cosmetic procedure because of their satisfactory results, less downtime, and limited side effects. However, some rare adverse events may occur soon after treatment. Here, we report on the case of a patient who developed a severe anaphylactic facial edema after botulinum toxin injections for frontal wrinkles.

\section{Case report}

A 28-year-old female visited our hospital for her first session of botulinum toxin injections to remove her frontal and glabella wrinkles. The patient denied a history of allergies to any food or drugs. One hundred units of botulinum toxin (CBTX-A; Lanzhou Institute of Biological Products, Lanzhou, China) was reconstituted in $2 \mathrm{ml}$ of $0.9 \%$ normal saline. No local anesthetic was administered topically or by injection. Botulinum toxin was injected in a total of 20 units divided into 2 units per site on the forehead. The injections were completed smoothly and uneventfully. Six hours post the injections, the patient's face started to swell up and redden with a burning feeling. By the following morning, her entire face had become swollen and she had a headache. The patient returned to the hospital with severe edema on her frontal forehead, eyelids, and cheeks without compromised swallowing or breathing problems (Fig. 1). She was diagnosed as experiencing an anaphylactic response to botulinum toxin after the level of immunoglobulin (Ig) E in

Received October 5, 2021; Revised November 12, 2021; Accepted November 13, 2021

Corresponding author: Chen Zhang

E-mail: neilzhang@sina.com

This is an Open Access article distributed under the terms of the Creative Commons Attribution Non-Commercial License (http://creativecommons.org/licenses/by-nc/4.0), which permits unrestricted non-commercial use, distribution, and reproduction in any medium, provided the original work is properly cited.

Copyright (C) 2021 Korean Society of Korean Cosmetic Surgery and Medicine (KSKCS \& KCCS). 


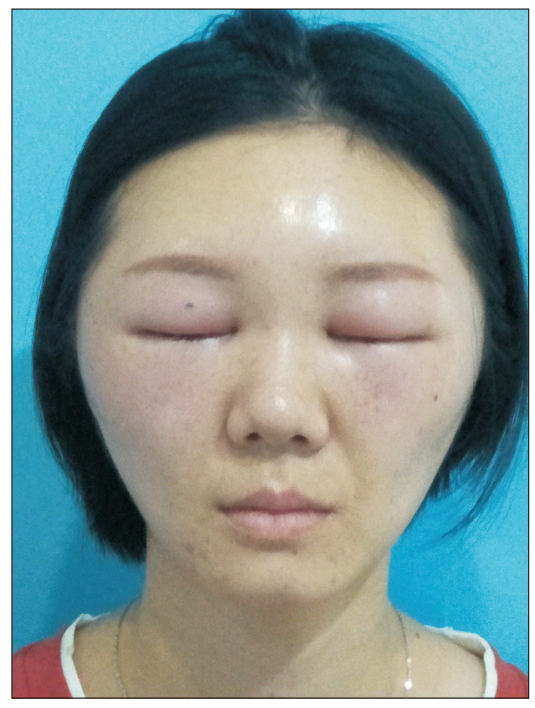

Fig. 1. The patient developed severe edema on her frontal head, eyelids, and cheeks after botulinum toxin injections.

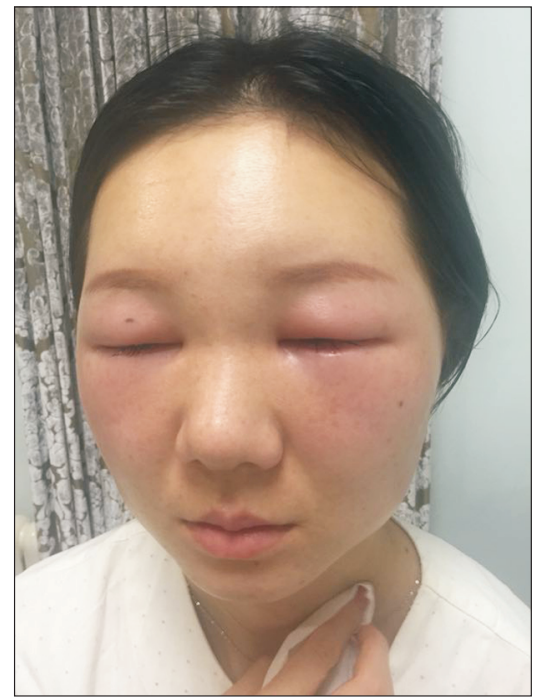

Fig. 2. Facial swelling worsened and extended to the neck one day after the botulinum toxin injections.

her blood serum was tested (the result was 1,658 IU/ml, much higher than the normal, 20-200 IU/ml), and given intravenous dexamethasone $20 \mathrm{mg}$ immediately. Oral oratadine was also prescribed once daily for a week. Unfortunately, her facial swelling worsened and extended to her neck by the evening of the same day (Fig. 2). An extra intravenous dexamethasone $20 \mathrm{mg}$ was administered to prevent potential laryngeal edema. Facial swelling began to resolve on the third morning. Intravenous dexamethasone was administered at $20 \mathrm{mg}$ and $10 \mathrm{mg}$ on the following 2 days. The residual swelling disappeared 7 days later (Fig. 3). The patient did not receive any further botulinum

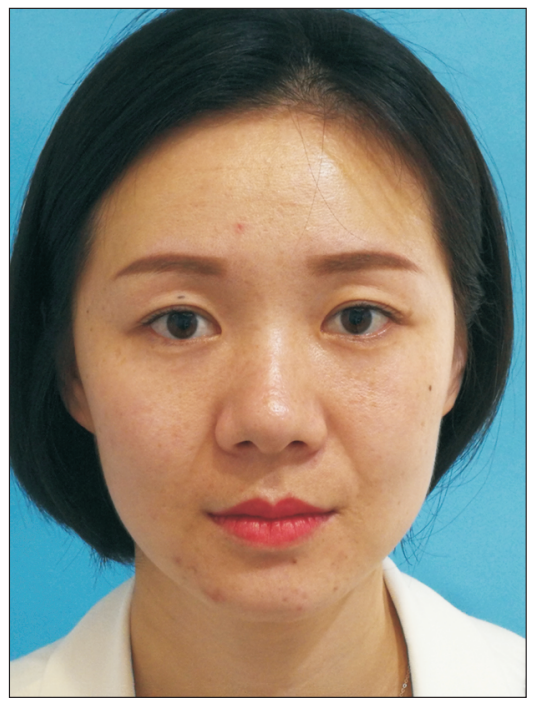

Fig. 3. The patient was cured 7 days after the botulinum toxin injections.

toxin injections, and no recurrence occurred during the 2-year follow-up period. The effect of BTXA was normal at the 3rd, 6th, and 24th month.

\section{Discussion}

There are few sporadic reports about anaphylaxis after botulinum toxin injections for frontal wrinkles. Rosenfield et al. [1] first reported a systemic reaction and confirmed an allergy to Botox administered at a cosmetic dose. The patient experienced severe itching after her fourth sequential injection of Botox and a second sequential injection of hyaluronic acid gel. She was diagnosed with a type IV hypersensitivity reaction using patch testing. Zhuang et al. [2] presented a patient with a hypersensitive skin rash after Botox injections for the treatment of facial wrinkles. The patient developed a whole-body skin rash, excluding the face and neck, 48 hours after the injections. She was diagnosed with Type I hypersensitivity after the level of IgE in her blood serum was tested. Careta et al. [3] reported a case of an allergic reaction to CBTX-A for wrinkles. The patient developed urticarial plaques proximal to the injection site, minutes after the application. Our case had different characteristics from those of the abovementioned incidents. This had been the patient's first time that she had received botulinum toxin injections at a cosmetic dose. Her mainly clinical manifestations were aggravated by a facial edema, as the level of IgE in her blood serum was much higher than normal. A consequence which had not previously been reported.

It should be noted that non-allergic, eyelid edema has been 
reported after botulinum toxin injections on the forehead. However, in that case, the antibodies were normal, and prick testing and patch testing were negative. Furthermore, the condition did not respond to anti-allergic treatment, usually effective for allergies. Eyelid edema is often caused by poor venous or lymphatic return to the eyelid, or decreased muscle tone. Periorbital edema due to botulinum toxin injections can be managed by the application of hot pads over the eyes, frequent blinking in the morning, and self-massage of the affected area to increase venous return. Preventive strategies include examination of the function and tone of the orbicularis oculi and levator palpebrae superioris muscles before treatment [4].

If anaphylactic edema is suspected after botulinum toxin injections, systemic corticosteroids and antihistamines should be administered to control the swelling. Should the patient not respond to the treatment, it is strongly recommended that he or she be referred to a nearby general hospital for further diagnosis and treatment.

It is not clear what factor associated with the botulinum toxin triggers an anaphylactic reaction in the body. It may be the toxin itself or the bovine gelatin, the carrier in the Chinese botulinum toxin, which has the potential to trigger an immunological response and allergic reaction [3].

In summary, this patient had facial edema after her first CBTX-A injection at a cosmetic dose and was controlled by the administration of antihistamines and systemic corticosteroids. This report is intended to caution doctors against possible adverse events in their everyday clinical work with botulinum toxin injections. As this is a report of a single case, further multicenter observations may be needed to determine its incidence.

\section{Conflicts of interest}

The author has nothing to disclose.

\section{References}

1. Rosenfield LK, Kardassakis DG, Tsia KA, Stayner G. The first case report of a systemic allergy to onabotulinumtoxinA (Botox) in a healthy patient. Aesthet Surg J 2014;34:766-8.

2. Zhuang Y, Yang MY, Liu CJ. [Whole body hypersensitive skin rash after Botox injection for treatment of facial wrinkles]. Chin J Aesthet Plast Surg 2015;26:255-6. Chinese.

3. Careta MF, Delgado L, Patriota R. Report of allergic reaction after application of botulinum toxin. Aesthet Surg J 2015;35:NP102-5.

4. Chang YS, Chang CC, Shen JH, Chen YT, Chan KK. Nonallergic eyelid edema after botulinum toxin type A injection: case report and review of literature. Medicine (Baltimore) 2015;94:e1610. 